\title{
Potencial de krigagem em modelos digitais de elevação para o planejamento da colheita florestal
}

\author{
Francisco de Assis Costa Ferreira ${ }^{1 *}$, Elton da Silva Leite ${ }^{2}$, Luis Carlos de Freitas ${ }^{3 *}$, Aline Pereira das Virgens ${ }^{3}$ \\ ${ }^{1}$ Veracel Celulose S/A. Rodovia BA 275, Km 24, CEP 45820-970, Eunápolis, BA, Brasil \\ 2Universidade Federal do Recôncavo da Bahia, Rua Rui Barbosa, 710, CEP 44380-000, Cruz das Almas, BA, Brasil \\ ${ }^{3}$ Universidade Estadual do Sudoeste da Bahia, Estrada do Bem Querer, Km 04, CEP 45031-300, Vitória da Conquista, BA, Brasil
}

"Autor correspondente:
assiscferreira@gmail.com

Termos para indexação:

Sensoriamento remoto

Corte

Extração

Index terms:

Remote sensing

Cutting

Extraction

Histórico do artigo:

Recebido em 29/08/2017

Aprovado em 11/12/2017

Publicado em 29/12/2017

doi: 10.4336/2017.pfb.37.92.1492
Resumo - Objetivou-se com este trabalho avaliar a krigagem em modelos digitais de elevação (MDEs) SRTM e ASTER como subsidio ao planejamento da colheita florestal. Foram testados os seguintes MDEs: SRTM com 30 m e com $90 \mathrm{~m}$ de resolução espacial (RE); ASTER com 30 m de RE; SRTM com 30 m e com 90 m krigado; ASTER 30 m krigado; e MDE padrão, obtido a partir de carta planialtimétrica. Com auxílio dos MDEs, foram gerados mapas de declividade, de acordo com as restrições operacionais de máquinas utilizadas na colheita florestal. Os MDEs foram avaliados por análise estatística descritiva e quali-quantitativa. Os MDEs apresentaram forte dependência espacial da altitude. As diferenças da raiz quadrada do erro médio entre os MDEs provenientes de imagens SRTM e ASTER originais apresentaram pequena discrepância em relação aos MDEs krigados. Os resultados da análise quantitativa e qualitativa evidenciaram melhora por meio da krigagem nos MDEs SRTM com $30 \mathrm{~m}$ e $90 \mathrm{~m}$ de RE. Entretanto, o MDE ASTER 30 m não apresentou o mesmo comportamento, sendo melhor em sua versão original. O MDE SRTM 30 m krigado apresentou maior potencial como subsidio ao planejamento da colheita florestal, na ausência de MDEs em maiores escalas.

\section{Kriging potential of digital elevation model for forest harvesting planning}

\begin{abstract}
The objective of this study was to evaluate kriging in SRTM and ASTER digital elevation models (DEMs) to assist in the planning of forest harvesting. The following DEMs were tested: SRTM with $30 \mathrm{~m}$ and with $90 \mathrm{~m}$ spatial resolution (SR); ASTER $30 \mathrm{~m}$ SR; krigged SRTM $30 \mathrm{~m}$ and $90 \mathrm{~m}$; krigged ASTER $30 \mathrm{~m}$; and standard MDE, obtained from a planialtimetric map. Using DEMs declivity maps were generated according to restrictions of harvesting machines in forest. DEMs were evaluated using descriptive statistical analysis, and qualitative and quantitative analysis. DEMs assessed presented significant altitude spatial dependence. The differences of root-mean-square error between DEMs from original SRTM and ASTER images showed little difference from krigged DEMs. The results of the quantitative and qualitative analysis showed improvement by means of kriging in SRTM DEMs with $30 \mathrm{~m}$ and $90 \mathrm{~m}$ of spatial resolution. However, DEM ASTER $30 \mathrm{~m}$ did not present the same behavior, showing better results in its original version. The krigged DEM SRTM $30 \mathrm{~m}$ presented more potential to be used in the forest harvest planning, when DEMs in larger scales are not available.
\end{abstract}




\section{Introdução}

Os modelos digitais de elevação (MDEs) são fundamentais em análises florestais, pois representam de maneira simples e de fácil entendimento a topografia do terreno (Aspiazú et al., 1990). A topografia precisa ser conhecida para a alocação adequada de máquinas florestais, as quais apresentam elevados custos, principalmente os de colheita florestal.

No planejamento da colheita florestal os MDEs são utilizados como subsidio para criação dos mapas de declividade, os quais indicam os locais que cada tipo de máquina florestal irá operar, de acordo com as especificações dos equipamentos, com reflexos na segurança, produtividade e custos de produção.

Os MDEs podem ser originados por vários processos, dentre eles os que demandam altos custos financeiros, como os levantamentos planialtimétricos por meio de receptores GNSS (Global Navigation Satellite System), estação total ou nível de luneta, ou obtidos de forma gratuita, por meio de imagens ASTER (Advanced Spaceborne Thermal Emissionand Reflection Radiometer) e SRTM (Shuttle Radar Topographic Mission).

A precisão dos MDEs derivados de sensores remotos é dependente da declividade do terreno, obtendo menor qualidade para áreas mais inclinadas (Gerstenecker et al., 2005). Neste contexto, a geoestatística, especificamente a krigagem, pode proporcionar melhorias na altimetria dos MDEs (Iwashita \& Souza Filho, 2009). Vieira (2000) cita que a krigagem minimiza as possibilidades de erros na espacialização dos dados, permitindo compreender e modelar com maior precisão a variabilidade espacial.

A avaliação de MDE pode ser realizada pela análise descritiva das áreas, especialmente pelo cálculo do erro quadrático médio (RMSE). Entretanto, Chagas et al. (2010) afirmam que o RMSE não deve ser utilizado isoladamente para avaliar a precisão vertical de MDE. Para auxiliar a avaliação do MDE, análises visuais podem ser utilizadas (Iwashita \& Souza Filho, 2009).

Tais análises também devem ser realizadas levandose em consideração a restrição de máquinas, de forma a estimar a melhor utilização das mesmas em função da declividade do terreno. Leite et al. (2014) comprovaram que a produtividade das máquinas é afetada pela declividade do terreno, podendo, em alguns casos, inviabilizar a mecanização florestal.
De forma geral, a comparação entre MDEs acessíveis gratuitamente, que permitam derivar de informações sobre a altitude, é de extrema relevância, permitindo conhecer potencialidades e restrições, além de contribuir para o planejamento e reduzir custos. Diante desse cenário, objetivou-se com este trabalho avaliar a krigagem em modelos digitais de elevação SRTM e ASTER visando subsidiar o gestor florestal no planejamento da colheita florestal.

\section{Material e métodos}

\section{Caracterização da área de estudo}

A área de estudo pertence ao município de Sardoá, MG. Possui 720,30 ha distribuídos em áreas com plantios florestais $(412,01 \mathrm{ha})$, estradas e aceiros $(43,56$ ha), reserva legal (208,76 ha) e áreas de preservação permanente $(55,97 \mathrm{ha})$. O mapa de uso do solo utilizado na pesquisa foi disponibilizado por uma empresa florestal da região.

\section{Obtenção dos modelos digitais de elevação}

Determinou-se um modelo digital de elevação (MDE padrão), que serviu como base para comparação com os modelos testados. O MDE padrão foi criado a partir de carta planialtimétrica com curvas de nível equidistantes $(10 \mathrm{~m})$, disponibilizadas por uma empresa florestal da região, geradas a partir de levantamento planialtimétrico com receptor GNSS geodésico de dupla frequência (L1 e L2). Utilizou-se a ferramenta topo to raster do software ArcGIS versão 10.2 para realizar o processamento do MDE de referência.

Os MDEs utilizados foram obtidos gratuitamente em meio digital, sendo estes: folha SE-23-Z-B, correspondente ao SRTM de $90 \mathrm{~m}$ de resolução espacial (RE); folha S19-W043, ao SRTM de $30 \mathrm{~m}$ de RE, e a folha S19-W043, correspondente à imagem ASTER (Advanced Spaceborne Thermal Emissionand Reflection Radiometer) de $30 \mathrm{~m}$ de RE.

Cabe ressaltar que os MDEs SRTM com RE de $30 \mathrm{~m}$ foram disponibilizados para o Brasil em 2015. Anteriormente a este período, os dados disponibilizados em meio digital apresentavam RE de 90 m (Grohmann et al., 2008).

A técnica de krigagem foi utilizada para otimizar os MDE SRTM e ASTER. Com o auxílio da ferramenta 
raster to point, os pixels dos MDEs foram convertidos em um shapefile de pontos, sendo posteriormente recortados de acordo com o perímetro da área em estudo.

Por meio destes pontos, foi gerado um semivariograma com o software GS+ (Robertson, 1998), conforme equação 1. O semivariograma foi utilizado para modelar a estrutura de variabilidade da altitude (z), analisado e ajustado de acordo com o melhor modelo matemático, com base nos parâmetros: patamar, alcance, efeito pepita, índice de dependência espacial (IDE) e coeficiente de determinação (Vieira, 2000).

$$
\left.Y(h)=\frac{1}{2 N(h)} x \sum_{i=1}^{N(h)}\left[Z\left(x_{i}\right)-x_{i}+h\right)\right]^{2}
$$

Em que: $\mathrm{Y}$ (h) é a semivariância em função da distância h; $\mathrm{N}$ (h) é o número de pares experimentais de observações $Z$ (xi) e $\mathrm{Z}(\mathrm{xi}+\mathrm{h})$ separados por uma distância $\mathrm{h}$.

O IDE foi classificado de acordo com a metodologia proposta por Zimback (2001), sendo a dependência espacial fraca para valores $\leq 25 \%$; moderada entre $25 \%$ e $75 \%$, e forte quando os valores são $\geq 75 \%$.

A interpolação foi realizada por meio da krigagem com auxílio do software ArcMap 10.2, de acordo com o modelo matemático estimado no software GS+, obtendose os seguintes modelos: SRTM $30 \mathrm{~m}$ krigado, SRTM $90 \mathrm{~m}$ krigado e ASTER $30 \mathrm{~m}$ krigado.

\section{Avaliações dos MDEs}

Os MDEs avaliados foram: SRTM 30 m; SRTM 90 m; ASTER 30 m; SRTM 30 m krigado; SRTM 90 m krigado; ASTER 30 m krigado, sendo comparados com o MDE padrão.

A avaliação da qualidade dos MDEs foi realizada por meio de análise estatística descritiva, considerando os seguintes parâmetros: valores mínimos, máximos, médios e raiz quadrada do erro médio (RMSE) das cotas altimétricas de 720 pontos de cada MDE.

Os pontos foram obtidos por meio de uma malha sistemática de pontos equidistante de $100 \mathrm{~m}$ em ambiente SIG. O cálculo do RMSE foi obtido conforme a equação 2 (Chagas et al., 2010).

$$
R M S E=\sqrt{\frac{\sum_{i=1}^{n}\left(D_{1}^{2}\right)}{n}}
$$

Em que: $D=$ diferença de elevação entre os MDE avaliados e o MDE padrão; $n=$ número de pontos da amostragem realizada.

\section{Restrições das máquinas de colheita florestal}

O planejamento da colheita foi estabelecido em função das restrições das faixas de declividade, divididas em três classes (Tabela 1), de acordo com a literatura (Oliveira Júnior et al., 2009; Simões \& Fenner, 2010; Burla et al., 2012; Fernandes et al., 2013; Leite et al., 2013, 2014; Robert et al., 2013).

A colheita florestal engloba várias atividades, destacando os processos de corte (derrubada, desgalhamento, destopamento e processamento) e extração (retirada da madeira do interior do talhão até a margem da estrada).

Tabela 1. Classes de declividade para as operações de colheita

\begin{tabular}{|c|c|c|}
\hline \multirow{2}{*}{$\begin{array}{l}\text { Classes de } \\
\text { declividade }\end{array}$} & \multicolumn{2}{|c|}{ Atividades da colheita florestal } \\
\hline & Corte & Extração \\
\hline $0^{\circ}$ a $15^{\circ}$ & $\begin{array}{l}\text { Sistema mecanizado: } \\
\text { máquinas adaptadas } \\
\text { (escavadeira hidráulica } \\
\text { adaptada com cabeçote } \\
\text { processador) e específicas } \\
\text { (harvester e feller-buncher) }\end{array}$ & $\begin{array}{l}\text { Sistema mecanizado: } \\
\text { máquinas adaptadas } \\
\text { (autocarregável) e } \\
\text { específicas (forwarder, } \\
\text { skkider, track skidder, } \\
\text { clambunk skidder) }\end{array}$ \\
\hline $15^{\circ}$ a $25^{\circ}$ & $\begin{array}{l}\text { Máquinas específicas } \\
\text { (harvester, feller-buncher) }\end{array}$ & $\begin{array}{l}\text { Máquinas específicas } \\
\text { (forwarder, skidder, } \\
\text { track skidder, clambunk } \\
\text { skidder) }\end{array}$ \\
\hline $25^{\circ}$ a $45^{\circ}$ & $\begin{array}{l}\text { Sistema semimecanizado } \\
\text { (motosserra) }\end{array}$ & $\begin{array}{l}\text { Equipamentos (guincho } \\
\text { arrastador, cabos aéreos e } \\
\text { guindastes). }\end{array}$ \\
\hline
\end{tabular}
florestal.

\section{Avaliação dos mapas de declividade gerados a partir dos MDEs}

A avaliação foi realizada de forma qualitativa e quantitativa. Considerou-se como avaliação qualitativa a correlação visual entre os MDEs SRTM e ASTER originais e, após a krigagem, em relação ao MDE padrão. Esse parâmetro permitiu identificar a presença de áreas discrepantes que podem interferir na colheita florestal, além de identificar visualmente os modelos que mais se assemelharam ao MDE padrão.

A avaliação quantitativa consistiu em mensurar as áreas por classe de declividade (Tabela 1) e a sobreposição das áreas dos MDEs avaliados em relação ao MDE padrão. Esse parâmetro permitiu identificar a quantidade de áreas que foram semelhantes entre os MDEs avaliados e o de referência. 


\section{Resultados}

Os parâmetros utilizados para determinação dos MDEs krigados obtidos a partir das imagens SRTM e ASTER estão apresentados na tabela 2. Observou-se que os MDEs apresentaram forte dependência espacial da altitude, com IDE maior que $75 \%$. O modelo esférico foi o que apresentou melhor ajuste.

Os resultados da análise estatística descritiva dos MDEs (Tabela 3), mostram diferenças entre SRTM, ASTER e imagens krigadas em relação ao MDE padrão.
Os mapas com as classes de declividade utilizadas no planejamento da colheita florestal consideradas no estudo estão apresentados na figura 1. Foram observadas discrepâncias entre os MDEs avaliados e o MDE de referência.

Pode-se observar na tabela 4 a sobreposição dos MDEs testados em relação ao MDE padrão. o MDE SRTM 30 m krigado apresentou maior semelhança de áreas com o MDE padrão (65,65\%) e o MDE ASTER 30 $\mathrm{m}$ krigado o modelo com menor porcentagem de áreas em comum $(54,76 \%)$.

Tabela 2. Parâmetro dos modelos digitais de elevação de imagens a partir da geoestatística.

\begin{tabular}{ccccccc}
\hline MDEs & Modelo ajustado & Patamar (Co+C) & Alcance & Efeito pepita (Co) & IDE (C/(Co+C)) & $\mathbf{R}^{2}$ \\
\hline SRTM 30 m krigado & Esférico & 2595 & 915 & 1 & $99,9 \%$ & $96,0 \%$ \\
ASTER 30 m krigado & Esférico & 2793 & 944 & 1 & $99,7 \%$ & $97,7 \%$ \\
SRTM 90 m krigado & Esférico & 2371 & 825 & 1 & $99,8 \%$ & $95,7 \%$ \\
\hline
\end{tabular}

IDE = índice de dependência espacial; $\mathrm{R}^{2}=$ coeficiente de determinação.

Tabela 3. Valores dos parâmetros dos modelos digitais de elevação (MDE).

\begin{tabular}{ccccc}
\hline MDEs & Valor mínimo (m) & Valor máximo $(\mathbf{m})$ & $\begin{array}{c}\text { Valor } \\
\text { médio }(\mathbf{m})\end{array}$ & RMSE $(\mathbf{m})$ \\
\hline MDE padrão & 724 & 954 & 819 & - \\
SRTM 30 m & 734 & 969 & 839 & 21 \\
SRTM 30 m krigado & 735 & 971 & 838 & 21 \\
ASTER & 735 & 966 & 834 & 18 \\
ASTER krigado & 737 & 959 & 833 & 17 \\
SRTM 90 m & 736 & 959 & 836 & 23 \\
\hline SRTM 90 m krigado & 736 & 957 & 837 & 24 \\
\hline RMSE = raiz quadrada do erro médio quadrático. & & &
\end{tabular}

Tabela 4. Sobreposição das classes de declividade dos modelos digitais de elevação testados em relação ao MDE padrão.

\begin{tabular}{|c|c|c|c|c|c|c|c|c|}
\hline \multirow{2}{*}{ MDE } & \multicolumn{8}{|c|}{ Quantificação das áreas por classe de declividade (ha) } \\
\hline & \multicolumn{2}{|c|}{$0-15^{\circ}$} & \multicolumn{2}{|c|}{$15-25^{\circ}$} & \multicolumn{2}{|c|}{$25-45^{\circ}$} & Total (ha) & Total $(\%)$ \\
\hline \multirow[t]{3}{*}{ MDE padrão } & \multicolumn{2}{|c|}{226,51} & \multicolumn{2}{|c|}{136,15} & \multicolumn{2}{|c|}{49,35} & 412,01 & $100,00 \%$ \\
\hline & \multicolumn{8}{|c|}{ Áreas de sobreposição entre os MDE testados e o MDE padrão } \\
\hline & ha & $\%$ & ha & $\%$ & ha & $\%$ & ha & $\%$ \\
\hline SRTM $30 \mathrm{~m}$ & 128,16 & 56,58 & 82,76 & 60,79 & 33,19 & 67,25 & 244,11 & 59,25 \\
\hline SRTM 30 m krigado & 175,80 & 77,61 & 77,94 & 57,25 & 16,75 & 33,94 & 270,49 & 65,65 \\
\hline ASTER $30 \mathrm{~m}$ & 157,83 & 69,68 & 70,40 & 51,71 & 18,83 & 38,16 & 247,06 & 59,96 \\
\hline ASTER $30 \mathrm{~m}$ krigado & 143,81 & 63,49 & 60,71 & 44,59 & 21,09 & 42,74 & 225,61 & 54,76 \\
\hline SRTM $90 \mathrm{~m}$ & 173,00 & 76,38 & 61,00 & 44,80 & 2,5 & 5,07 & 236,5 & 57,40 \\
\hline SRTM 90 m krigado & 174,54 & 77,06 & 68,70 & 50,46 & 9,87 & 20,00 & 253,11 & 61,43 \\
\hline
\end{tabular}



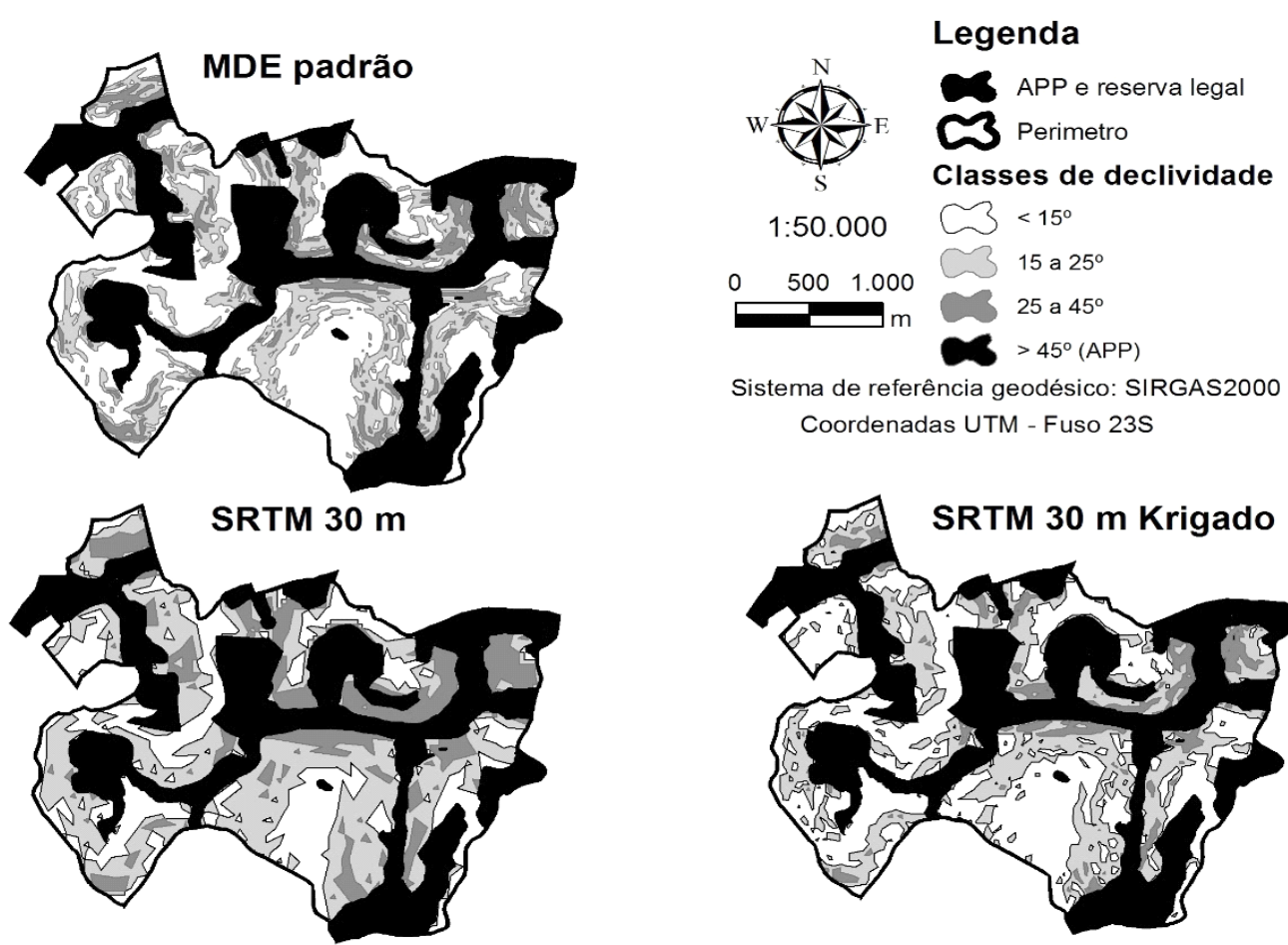

Sistema de referência geodésico: SIRGAS2000

Coordenadas UTM - Fuso $23 S$
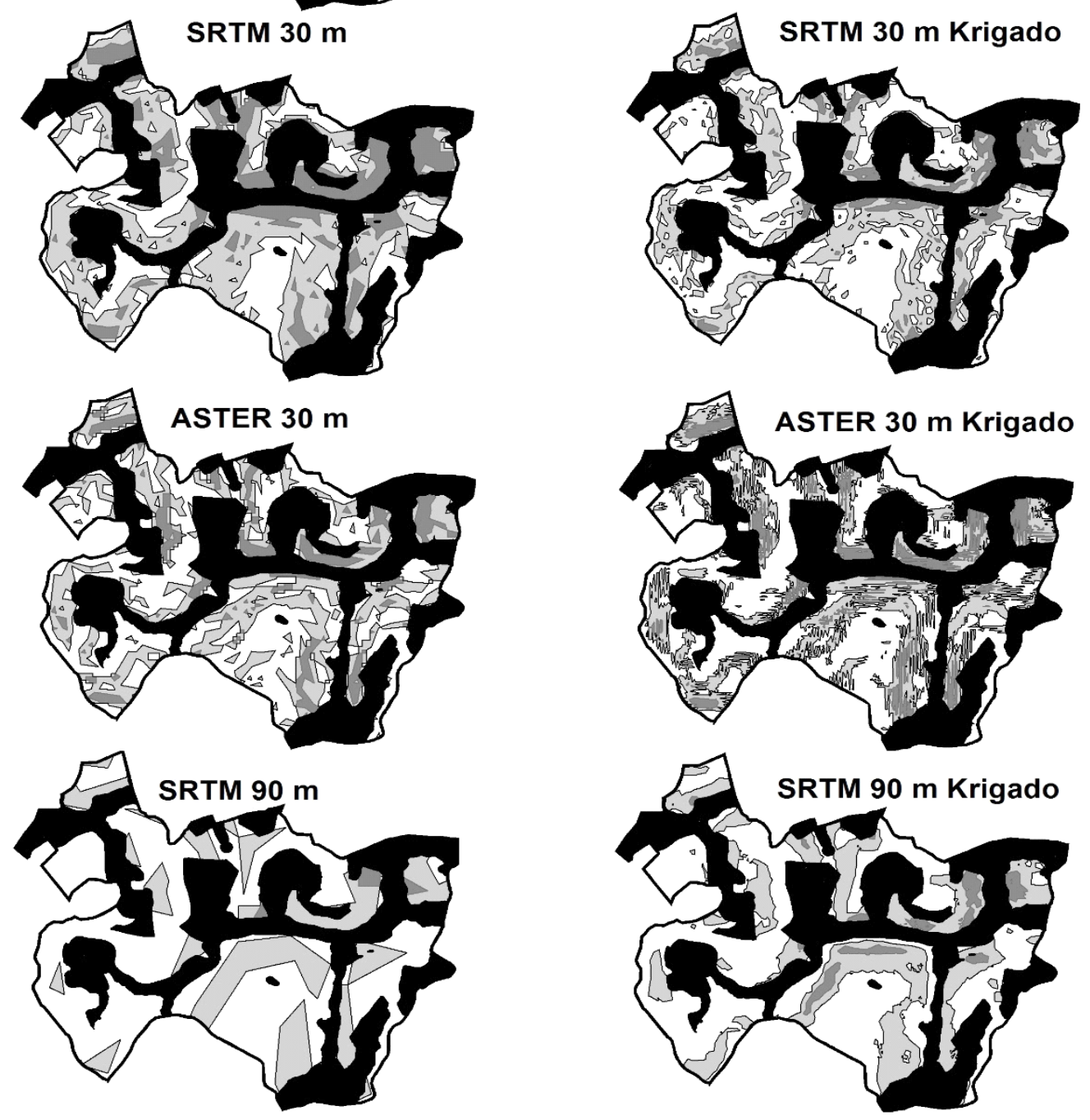

Figura 1. Mapas de declividade de acordo com as classes utilizadas no estudo. 


\section{Discussão}

Observa-se elevada semelhança entre os valores de RMSE produzidos pelos MDE ASTER $30 \mathrm{~m}(18 \mathrm{~m}) \mathrm{e}$ ASTER krigado $30 \mathrm{~m}(17 \mathrm{~m})$ (Tabela 3$)$. Hirano et al. (2003) afirmam que valores adequados do RMSE para o sensor ASTER estão entre 7 (sete) e $15 \mathrm{~m}$. De acordo com os mesmos autores, ambos podem ser alcançados desde que utilizados softwares adequados, bem como imagens de boa qualidade e pontos de controle terrestres.

Os MDEs SRTM 30 m, SRTM 30 m krigado, SRTM $90 \mathrm{~m}$ e SRTM $90 \mathrm{~m}$ krigado apresentaram valores superiores de RMSE, quando comparados aos MDEs provenientes do sensor ASTER. Barros et al. (2005) obtiveram valor do RMSE de 23,94 m para um MDE SRTM 90 m de uma área litorânea montanhosa no sul do Estado do Rio de Janeiro, resultado semelhante ao do presente estudo, que também apresenta característica montanhosa.

Chagas et al. (2010) compararam por meio do critério RMSE a precisão vertical de MDE SRTM $90 \mathrm{~m}$ (12 m) e ASTER $30 \mathrm{~m} \mathrm{(37} \mathrm{m),} \mathrm{identificando} \mathrm{diferença}$ de $25 \mathrm{~m}$ entre os modelos. Os autores citaram que em áreas com elevadas variações de altimetria, como no estudo desenvolvido pelos autores, podem haver valores de RMSE discrepantes. No presente estudo, os valores apresentaram-se próximos, com diferença de $5 \mathrm{~m}$ entre o sensor ASTER $(18 \mathrm{~m})$ e SRTM $90 \mathrm{~m}$ $(23 \mathrm{~m})$, principalmente em função do estudo ter considerado uma área de abrangência pequena (412 ha) quando comparado a outros trabalhos, como o de Chagas et al. (2010), os que trabalharam em uma área de 28.000 ha.

As diferenças do RMSE entre os MDEs provenientes de imagens SRTM e ASTER apresentaram pequena discrepância em relação aos MDEs krigados, obtendo-se valores máximos, mínimos e médios semelhantes entre os modelos (tabela 3). Em relação ao MDE padrão, os resultados do RMSE estimados pelos MDE originais e krigados variaram entre 17 e $24 \mathrm{~m}$. Esta diferença pode ser atribuída à forma de aquisição dos dados das imagens, pois a interferometria (SRTM) e esteroscopia (ASTER) podem utilizar a vegetação arbórea como parâmetro para determinar a altitude. Tomazoni et al. (2011) encontraram resultados semelhantes ao avaliarem MDE a partir de imagens ASTER e SRTM. Os autores destacaram que a vegetação influencia na altitude dos modelos, justificando as discrepâncias em relação à carta planialtimétrica.
Os resultados da Tabela 3 evidenciaram que os valores máximos, mínimos, médios e a RMSE não foram suficientes para avaliar a precisão de modelos digitais de elevação, o que, segundo Chagas et al. (2010), requereria outros tipos de análise, como análise qualitativa de MDE.

O planejamento da colheita florestal em relevo com muitas variações de altitude deve ser criterioso, para atender às restrições de tráfego de máquinas, justificando o investimento em bases planialtimétricas de alta precisão. Leite et al. $(2013,2014)$ avaliaram o desempenho do harvester e do forwarder, respectivamente, e afirmaram que quanto maior a declividade, menor a produtividade das máquinas florestais.

Oliveira Júnior et al. (2009) avaliaram a produtividade de feller-buncher em povoamento de eucalipto em relevo acidentado, relatando que a operação de colheita foi significativamente influenciada pela inclinação do terreno em qualquer padrão de floresta. Birro et al. (2002), avaliando a produtividade do track skidder em diferentes classes de declividade, constataram a influência do relevo na produtividade da extração florestal.

Assim, quanto mais preciso for o MDE, melhor será o planejamento da colheita florestal e menores serão os custos em virtude da alocação correta das máquinas, bem como do aumento da segurança operacional. $\mathrm{Na}$ ausência de MDEs em grandes escalas, que permitam maior nível de detalhamento do relevo, a utilização de base de dados gratuito pode ser uma alternativa, desde que seja do conhecimento de seus usuários suas reais potencialidades e restrições.

A maior semelhança do MDE SRTM $30 \mathrm{~m}$ krigado em relação ao MDE padrão, principalmente na classe de $0^{\circ}$ a $15^{\circ}$, evidencia melhorias nas áreas mais planas, passando de $56,58 \%$ para $77,61 \%$ de sobreposição, representando adequação de 47,64 ha (Figura 1, Tabela 4). O MDE ASTER krigado $30 \mathrm{~m}$ apresentou resultado negativo nessa análise, reduzindo o percentual de $69,68 \%$ para $63,49 \%$, o que correspondeu a uma área de 9,69 ha.

Na classe intermediária $\left(15^{\circ}\right.$ a $\left.25^{\circ}\right)$, o MDE SRTM $30 \mathrm{~m}$ krigado apresentou pequena redução $(3,54 \%) \mathrm{em}$ relação ao MDE SRTM 30 m; e o ASTER krigado 30 m apresentou $7,12 \%$ de redução em comparação ao MDE ASTER $30 \mathrm{~m}$. Nas áreas mais inclinadas $\left(25^{\circ}\right.$ a $\left.45^{\circ}\right)$, o SRTM $30 \mathrm{~m}$ krigado apresentou maiores reduções de percentagem em relação ao SRTM 30 m (33,31\%), o que pode ser explicado pela adequação das áreas de declividade entre $0^{\circ}$ e $15^{\circ}$, já que a porcentagem total 
de sobreposição em relação ao MDE padrão passou de 59,25 \% para 65,65\% (adequação de 26,38 ha). Em contrapartida, na declividade de $25^{\circ}$ a $45^{\circ}$, observou-se pequena melhoria para o MDE ASTER krigado $30 \mathrm{~m}$ $(4,62 \%)$.

Em relação ao MDE SRTM $90 \mathrm{~m}$ krigado, o MDE apresentou melhor desempenho nas áreas mais inclinadas, passando à sobreposição de $44,80 \%$ a 50,46\% na classe de 15 a $25^{\circ}$ e de 5,07\% para $20,00 \%$ na classe de 25 a $45^{\circ}$. O MDE SRTM 90 m krigado apresentou melhorias mais expressivas e aproximação visual com o MDE padrão, mostrando ainda melhor desempenho em relação ao modelo original da imagem.

Os resultados da Tabela 4 analisados de forma conjunta com a Figura 1 evidenciaram que o MDE SRTM 30 m krigado apresentou melhor aproximação da superfície em comparação ao MDE estabelecido como padrão neste estudo, indicando que, na ausência de um planejamento em maior escala, esse MDE apresenta potencial para realizar planejamento da colheita florestal, principalmente em áreas mais planas. O MDE ASTER $30 \mathrm{~m}$ também apresentou muitas semelhanças com o MDE padrão. Entretanto, o mapa gerado com este MDE apresentou muitos polígonos que não ocorreram no $\mathrm{MDE}$ padrão. Este aspecto pode influenciar negativamente as operações mecanizadas.

\section{Conclusão}

A utilização da krigagem melhorou a qualidade dos MDEs SRTM, configurando como uma alternativa para o planejamento da colheita florestal no que se refere à alocação de máquinas em cada situação de declividade, com reflexos positivos na segurança e rendimento das operações.

A avaliação qualitativa e quantitativa do MDE SRTM $30 \mathrm{~m}$ krigado apresentou valores mais semelhantes ao MDE padrão, quando comparado aos outros MDE testados, demonstrando maior potencial de uso quando da ausência de base de dados planialtimétricos em escalas maiores.

Como a avaliação foi realizada em áreas de relevo extremamente acidentado, recomenda-se que análise semelhante seja feita em locais com menor incidência de áreas inclinadas.

\section{Referências}

Aspiazú, C. et al. Modelos digitais de terrenos conceituação e importância. Boletim de Pesquisa Florestal, n. 21, p. 27-36, 1990.

Barros, R. S. et al. Avaliação do modelo digital de elevação do SRTM na ortorretificação de imagens Landsat 7 - Área de aplicação: Angra dos Reis, RJ. In: SIMPÓSIO BRASILEIRO DE SENSORIAMENTO REMOTO, 12., 2005, Goiânia. Anais... São José dos Campos: INPE, 2005. p. 3997-4004.

Birro, M. H. B. et al. Avaliação técnica e econômica da extração de madeira de eucalipto com "track-skidder" em região montanhosa. Revista Árvore, v. 26, n. 5, p. 525-532, 2002. DOI: 10.1590/S010067622002000500001 .

Burla, E. R. et al. Avaliação técnica e econômica do harvester em diferentes condições operacionais. Engenharia na Agricultura, v. 20, p. 412-422, 2012.

Chagas, C. S. et al. Avaliação de modelos digitais de elevação para aplicação em um mapeamento digital de solos. Revista Brasileira de Engenharia Agrícola e Ambiental, v. 14, n. 2, p. 218 226, 2010. DOI: 10.1590/S1415-43662010000200014.

Fernandes, H. C. et al. Avaliação técnica e econômica de um "Harvester" em diferentes condições de terreno e produtividade da Floresta. Scientia Forestalis, v. 41, n. 97, p. 145-151, 2013.

Gerstenecker, C. et al. Validation of digital elevation models around Merapi Volcano Java, Indonesia. Natural Hazards and Earth System Sciences, v. 5, p. 863-876, 2005. DOI: 10.5194/ nhess-5-863-2005.

Grohmann, C. H. et al. Aplicações dos modelos de elevação SRTM em geomorfologia. Revista Geográfica Acadêmica, v. 2, n. 2. p. 73-78, 2008.

Hirano, A. et al. Mapping from ASTER stereo image data: DEM validation and accuracy assessment. ISPRS Journal of Photogrammetry\& Remote Sensing, v. 57, p. 356-370, 2003. DOI: 10.1016/S0924-2716(02)00164-8.

Iwashita, F. \& Souza Filho, C. R. de. Avaliação da interpolação de dados SRTM $90 \mathrm{~m}$ através de dados SRTM $30 \mathrm{~m}$ do território americano. In: SIMPÓSIO BRASILEIRO DE SENSORIAMENTO REMOTO, 14., 2009, Natal. Anais... São José dos Campos: INPE, 2009. p. 3927-3934.

Leite, E. S. et al. Modelagem do desempenho da extração de madeira pelo "forwarder". Revista Árvore, v. 38, n. 5, p. 879-887, 2014. DOI: $10.1590 / \mathrm{S} 0100-67622014000500012$.

Leite, E. S. et al Modelagem técnica e de custos do Harvester no corte de madeira de eucalipto no sistema de toras curtas. Scientia Forestalis, v. 41, n. 98, p. 205-215, 2013.

Oliveira Júnior, E. et al. Produtividade de feller-buncher em povoamento de eucalipto em relevo acidentado. Floresta, v. 39, n. 4, p. 905-912, 2009. DOI: 10.5380/rf.v39i4.16327.

Robert, R. C. G. et al. Avaliação do desempenho operacional do harvester 911.3 X3M em áreas declivosas. Floresta e Ambiente, v. 20, n. 2, p. 183-190, 2013. DOI: 10.4322/floram.2013.011. 
Robertson, G. P. GS+: geostatistics for the environmental sciences (version 5.1 for windows). [S. 1.]: Gamma Design Soffware, 1998. $152 \mathrm{p}$.

Simões, D. \& Fenner, P. T. Influência do relevo na produtividade e custos do "harvester". Scientia Forestalis, v. 38, n. 85, p. 107-114, 2010 .

Tomazoni, J. C. et al. Uso de modelo digital de elevação gerado a partir do ASTER GDEM e SRTM para caracterização de rede de drenagem do município Renascença no Sudoeste do Estado do Paraná. Revista Brasileira de Geografia Física, v. 4, n. 2, p. 365 $376,2011$.
Vieira, S. R. Geoestatística em estudos de variabilidade espacial do solo. In: Novais, R. F. et al. Tópicos em ciência do solo. Viçosa, MG: Sociedade Brasileira de Ciência do Solo, 2000. v. 1. p. 1-54.

Zimback, C. R. L. Análise espacial de atributos químicos de solos para fins de mapeamento da fertilidade. 2001. $114 \mathrm{f}$. Tese (LivreDocência em Levantamento do Solo e Fotopedologia) - Universidade Estadual Paulista, Botucatu. 